\title{
Magnetic Field Dependence of Excitations Near Spin-Orbital Quantum Criticality
}

\author{
A. Biffin, ${ }^{1,2}$ Ch. Rüegg, ${ }^{1,3}$ J. Embs, ${ }^{1}$ T. Guidi, ${ }^{4}$ D. Cheptiakov, ${ }^{1}$ A. Loidl, ${ }^{5}$ V. Tsurkan,${ }^{5,6}$ and R. Coldea ${ }^{2}$ \\ ${ }^{1}$ Laboratory for Neutron Scattering and Imaging, Paul Scherrer Institut, 5232 Villigen, Switzerland \\ ${ }^{2}$ Clarendon Laboratory, University of Oxford, Parks Road, Oxford OX1 3PU, United Kingdom \\ ${ }^{3}$ Department of Quantum Matter Physics, University of Geneva, 1211 Geneva, Switzerland \\ ${ }^{4}$ ISIS Facility, Rutherford Appleton Laboratory, Chilton, Didcot OX11 OQX, United Kingdom \\ ${ }^{5}$ Experimental Physics 5, Center for Electronic Correlations and Magnetism, Institute of Physics, \\ University of Augsburg, D-86159 Augsburg, Germany \\ ${ }^{6}$ Institute of Applied Physics, Academy of Sciences of Moldova, MD-2028 Chisinau, Republic of Moldova
}

(Received 30 June 2016; revised manuscript received 8 November 2016; published 10 February 2017)

\begin{abstract}
The spinel $\mathrm{FeSc}_{2} \mathrm{~S}_{4}$ has been proposed to realize a near-critical spin-orbital singlet (SOS) state, where entangled spin and orbital moments fluctuate in a global singlet state on the verge of spin and orbital order. Here we report powder inelastic neutron scattering measurements that observe the full bandwidth of magnetic excitations and we find that spin-orbital triplon excitations of an SOS state can capture well key aspects of the spectrum in both zero and applied magnetic fields up to $8.5 \mathrm{~T}$. The observed shift of low-energy spectral weight to higher energies upon increasing applied field is naturally explained by the entangled spin-orbital character of the magnetic states, a behavior that is in strong contrast to spin-only singlet ground state systems, where the spin gap decreases upon increasing applied field.
\end{abstract}

DOI: 10.1103/PhysRevLett.118.067205

When magnetic ions possess an orbital degeneracy in addition to spin, the combined effects of the on-site spin-orbit coupling and the intersite magnetic exchange interactions have been theoretically proposed to stabilize correlated states with entangled spin-orbital character and novel quasiparticles $[1,2]$. Generally such physics is not directly experimentally accessible as symmetry-lowering Jahn-Teller (JT) structural distortions [3] tend to lift orbital degeneracy leaving a spin-only degree of freedom. However, in the case of relatively strong spin-orbit coupling, or certain crystal structures where JT distortions are inhibited by the lattice geometry, spin-orbit entanglement can become manifest. For $d^{4}$ [2] and $d^{6}$ [4] transition metal ions in certain highsymmetry crystal environments the single-ion ground state is a spin-orbit entangled $J_{\text {eff }}=0$ singlet with an excited $J_{\text {eff }}=1$ triplet at higher energy. In this case, a theoretically proposed phase diagram [1] as a function of the ratio $x$ of magnetic exchange couplings to the singlet-triplet gap $\lambda$ is shown in Fig. 1. Cooperative spin and orbital order is expected for $x>x_{c}$, with a novel amplitude ("Higgs") mode for $x \gtrsim x_{c}$ [2,5] and entangled spin-orbital fluctuations present at the critical point $x_{c}$. In the regime of moderate exchange interactions, $x \lesssim x_{c}$, spins and orbitals are expected to be strongly fluctuating in a quantum paramagnetic state denoted as a "spin-orbital singlet" (SOS), with strong correlations between sites [1]. Even though the SOS state has no spin or orbital order, it supports quasiparticles, so called "spin-orbital triplons" (or "spin orbitons" [6]), corresponding to isotropically polarized, spin and orbital density wave packets that can propagate coherently across the lattice.

The spinel $\mathrm{FeSc}_{2} \mathrm{~S}_{4}$ has been proposed $[1,7,8]$ as a unique candidate to display a SOS state with intermediate-strength exchange interactions $\left(x \lesssim x_{c}\right)$ that bring it almost on the verge of spin and orbital order. It is the only known system to explore the physics of highly dispersive spin-orbital triplons, that may be close to spin-orbital quantum criticality. Here we report inelastic neutron scattering (INS) measurements over the full bandwidth of the magnetic excitations and we find good agreement with the expected spectrum of spin-orbital triplons of a near-critical SOS state. In applied magnetic field we observe a striking shift of the low-energy spectral weight to higher energies, a direct fingerprint of the entangled spin-orbital character of the magnetic states.

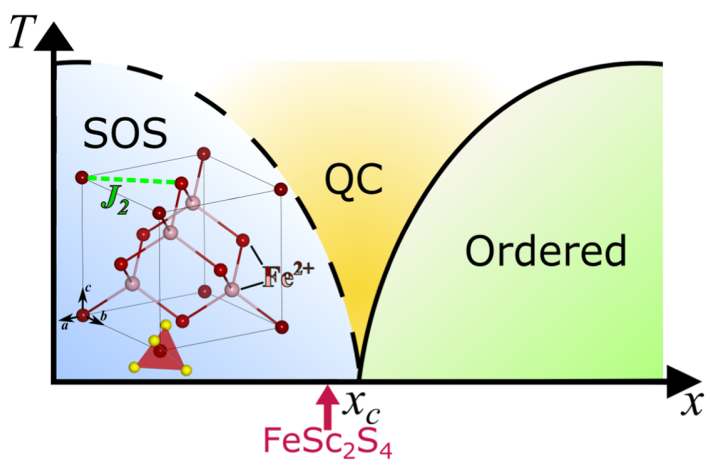

FIG. 1. Generic phase diagram as a function of $x=J_{2} / \lambda[1]$ with location of $\mathrm{FeSc}_{2} \mathrm{~S}_{4}$ indicated by the vertical arrow. Labels SOS, QC, and Ordered stand for spin-orbital singlet, quantum critical, and spin and orbitally ordered, respectively. Solid (dashed) line indicates a phase transition boundary (crossover). Inset shows $\mathrm{Fe}^{2+}$ ions in $\mathrm{FeSc}_{2} \mathrm{~S}_{4}$ are locally coordinated by a regular tetrahedron (red shaded polyhedron) of $\mathrm{S}^{2-}$ atoms (yellow balls) and are arranged in two FCC sublattices (light and dark red balls) with intrasublattice NN AFM exchange $J_{2}$. 
$\mathrm{FeSc}_{2} \mathrm{~S}_{4}$ has a cubic crystal structure with space group $F d \overline{3} m$ (no. 227) and lattice parameter $a=10.51 \AA$ at $300 \mathrm{~K}[9] . \mathrm{Fe}^{2+}$ ions are tetrahedrally coordinated by $\mathrm{S}^{2-}$ and in this crystal field of cubic symmetry the one-electron $d$ orbital states of $\mathrm{Fe}^{2+}$ are split into a lower $e$ doublet and upper $t_{2}$ triplet. Hund's coupling stabilizes a high-spin $(S=2)$ state, $e^{3} t_{2}^{3}$, with a twofold orbital degeneracy. The atomic spin-orbit interaction $\lambda_{0} \boldsymbol{L} \cdot \boldsymbol{S}$ lifts this twofold orbital and fivefold spin degeneracy to stabilize a SOS ground state with wave function [4]

$$
\frac{1}{\sqrt{2}}\left|3 z^{2}-r^{2}\right\rangle|0\rangle+\frac{1}{2}\left|x^{2}-y^{2}\right\rangle(|-2\rangle+|+2\rangle),
$$

where for each term the first ket gives the (multielectron) orbital state and the second ket the $S_{z}$ eigenvalue. The first excited state is a triplet above a gap $\lambda$ and local singlettriplet transitions then form the key ingredient from which coherently propagating triplons develop in the presence of intersite exchange interactions.

Previous susceptibility, specific heat, and NMR measurements on $\mathrm{FeSc}_{2} \mathrm{~S}_{4}[7,10]$ showed no clear anomalies indicative of spin or orbital order in spite of strong magnetic interactions manifested by a large antiferromagnetic (AFM) Curie-Weiss temperature of $-45 \mathrm{~K}$, indicating that the material may indeed be in the SOS phase. INS studies [8] focusing on the very low energy dynamics indicated that the dominant magnetic interaction is an AFM exchange $J_{2}$ between spins located at next-nearest neighbor (NNN) sites. This splits the diamond lattice into two magnetically decoupled, frustrated FCC lattices (light and dark sites in Fig. 1), where $J_{2}$ acts on NN bonds.

We have probed the magnetic excitations using INS measurements first in zero magnetic field and at temperatures $2.2-50 \mathrm{~K}$, using the direct-geometry, time-of-flight spectrometer MERLIN at the ISIS neutron source [11]. The sample was a $4 \mathrm{~g}$ powder of $\mathrm{FeSc}_{2} \mathrm{~S}_{4}$ synthesized as described in Ref. [12] and used in previous thermodynamic and diffraction studies [7]. The INS intensities were converted into absolute units by normalization to data measured on a vanadium standard. For incident neutrons of energy $E_{i}=15 \mathrm{meV}$ the covered phase space observed the full bandwidth of magnetic excitations, which showed prominent dispersions with a bandwidth extending to around $4 \mathrm{meV}$ at the lowest temperatures, as shown in Fig. 2(a). The high-temperature data were used to parametrize and subtract the nonmagnetic background (as described in Ref. [12]), such that Fig. 2(a) shows the magnetic signal only. Within experimental uncertainty no additional magnetic transitions were detected at higher energy transfers (data collected using incident neutron energies up to $200 \mathrm{meV}$ ). This is consistent with the expectation that the single-ion ground state is close to the SOS wave function in Eq. (1), for which no other (crystal-field) transitions are symmetry allowed [12]. In
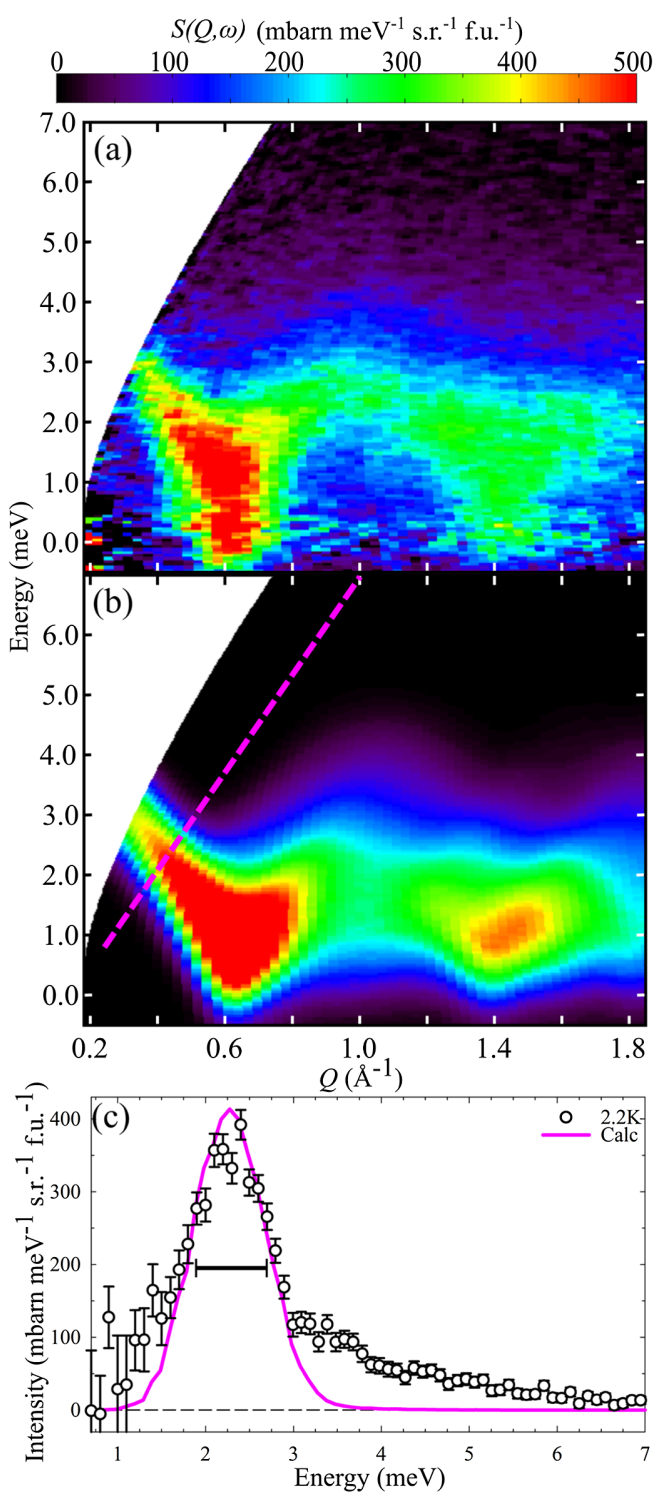

FIG. 2. (a) Background subtracted (see Ref. [12]) zero field INS data observing highly dispersive magnetic excitations (MERLIN, $E_{i}=15 \mathrm{meV}, 12 \mathrm{~h}$ counting time, energy resolution $0.79 \mathrm{meV}$ FWHM on the elastic line). (b) Corresponding one-triplon spectrum including convolution with the instrumental resolution. (c) Intensity along the tilted dashed line direction in (b), compared to the model calculation (solid line). Horizontal bar indicates expected peak FWHM due to instrumental resolution and spherical averaging.

agreement with previous low-energy studies [8], we observe a softening of the magnetic excitations near a critical wave vector $Q_{S} \approx 0.6 \AA^{-1}$ [see Fig. 2(a)], whose magnitude coincides with the structurally forbidden (100) reciprocal lattice position (in units of $2 \pi / a$ ) and a natural wave vector for AFM ordering on the fcc lattice [1]. Higher-resolution measurements shown in Fig. 3(a) indicate a clear suppression of scattering weight below $\sim 0.4 \mathrm{meV}$, indicating that the gap is much smaller than the full bandwidth of the magnetic excitations extending to 


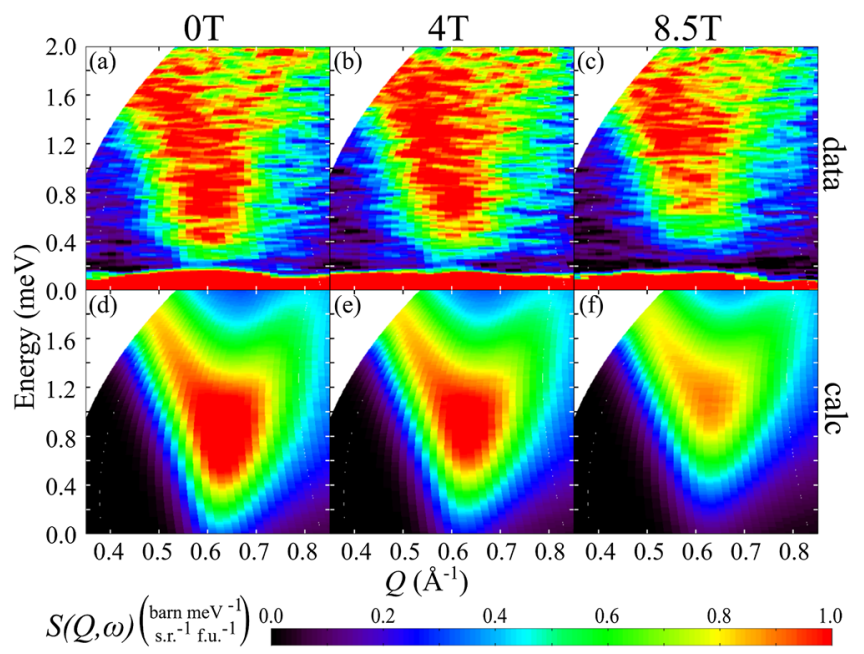

FIG. 3. (a)-(c) Background-subtracted INS data at 0, 4, and 8.5 T magnetic field compared with model calculations (d)-(f), respectively. The data was collected using FOCUS with $E_{i}=3.27 \mathrm{meV}$, elastic line energy resolution $0.18 \mathrm{meV}$ FWHM and $11 \mathrm{~h}$ counting per setting.

around $4 \mathrm{meV}$. This is consistent with the proposal that $\mathrm{FeSc}_{2} \mathrm{~S}_{4}$ is in the very close proximity of the critical point between SOS and magnetic or orbital order, at which the gap would be expected to close [1].

The magnetic field dependence of the excitations was measured on the same powder sample using the FOCUS time-of-flight spectrometer at the Swiss Spallation Neutron Source SINQ (PSI) with the sample placed inside a vertical 9 T cryomagnet. The obtained magnetic INS signal is plotted in Figs. 3(a)-3(c). By comparing the data at different fields it is apparent that the intense $V$-shaped magnetic signal near $Q_{S}$ shifts upwards upon increasing field. This trend is directly seen in the energy scan in Fig. 4(a) by comparing the data at 0,4 , and $8.5 \mathrm{~T}$; the spectral weight moves to higher energies upon increasing field.

Below we compare quantitatively the dispersive features of the excitation spectrum and the observed magnetic field dependence of the low-energy scattering with a model of spin-orbital triplons of a SOS ground state. In zero field the triplon dispersion derived in the harmonic approximation using pseudoboson operators [12] or alternative methods $[21]$ is

$$
\hbar \omega(\boldsymbol{k})=\lambda \sqrt{1+\frac{4 J(\boldsymbol{k})}{\lambda}},
$$

where $J(\boldsymbol{k})=J_{2} \sum_{\boldsymbol{A}} \cos (\boldsymbol{k} \cdot \boldsymbol{A})$ is the Fourier transform of the exchange couplings and $\boldsymbol{A}$ runs over all NN vectors of an FCC sublattice. The local singlet-triplet gap $\lambda$ is determined by the crystal field strength parametrized (using standard convention [22]) by the single parameter $B_{4}<0$, and the atomic spin-orbit coupling $\lambda_{0}<0$. Within a minimal $\left(J_{2}, B_{4}, \lambda_{0}\right)$ model we calculate the powder-averaged INS

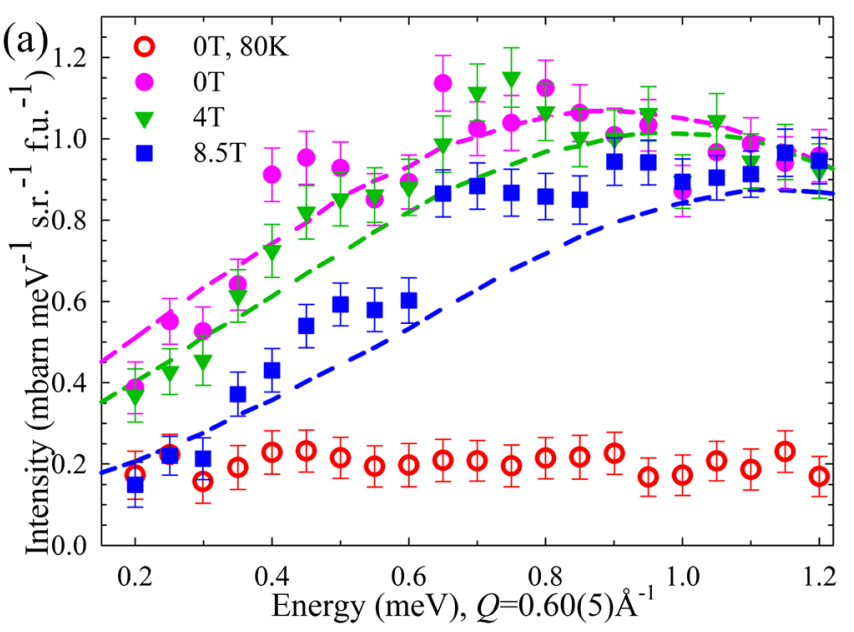

(b)

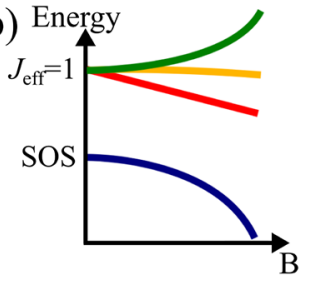

(c)

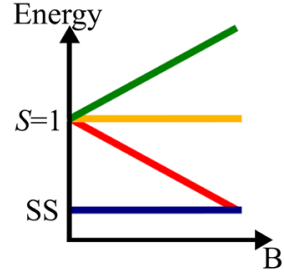

FIG. 4. (a) Energy scan at the softening wave vector $Q_{S}$ through the background subtracted data in Figs. 3(a)-(c): filled circles, triangles, and squares denote fields of 0,4 , and $8.5 \mathrm{~T}$, respectively. Dashed lines are the model calculations shown in Figs. 3(d)-(f). Data at $0 \mathrm{~T}$ in the paramagnetic phase at high temperatures $(80 \mathrm{~K})$ are also included (red open circles). (b) Schematic of the ground and excited states' energies as a function of applied field in the case of a SOS and (c) a spin-singlet system.

spectrum including the triplons' dynamical structure factor (for details see Ref. [12]) and compare systematically with scans through the INS data as shown in Figs. 2(c) and 4(a) (magenta filled symbols). In addition, we require the model parameters to reproduce optical data: the sharp $4.46 \mathrm{meV}$ absorption in THz spectroscopy [6,23], identified with the triplon energy (2) at the zone center, $\hbar \omega(0)=\sqrt{\lambda\left(\lambda+48 J_{2}\right)}$, and the sharp optical absorption at $\hbar \Omega=262(2) \mathrm{meV}$, attributed to the transition from the ground state to the lowest level of the upper orbital triplet [24]. Using those multiple constraints the best fit parametrization is obtained for $J_{2}=0.136(6), B_{4}=-2.45(6)$, and $\lambda_{0}=-12.1(1) \mathrm{meV}$, which give $\lambda=2.28(6) \mathrm{meV}$ [26]. This parametrization reproduces (by construction) the energies of both optical transitions and the $J_{2}$ value is comparable to that deduced from Curie-Weiss fits of the high-temperature susceptibility [23] and estimated from density-functional calculations [27]. The $B_{4}$ and $\lambda_{0}$ values are comparable with -2.58 and $-10.0(9) \mathrm{meV}$, respectively, found for $\mathrm{Fe}^{2+}$ ions in $\mathrm{FeCr}_{2} \mathrm{~S}_{4}$ [28].

The INS spectrum for the fitted parameter values is shown in Fig. 2(b), where we have also included an intrinsic linewidth broadening $\sim 1 \mathrm{meV}$, a possible significance of this broadening will be discussed later. The parametrization 
by the minimal model captures well the key features of the INS data with clear $V$-shaped dispersions and mode softenings near 0.6 and $1.4 \AA^{-1}$, identified with scattering emanating near the reciprocal lattice positions (100) and (211), respectively. A corresponding calculation performed for the data measured on FOCUS at zero field is shown in Fig. 3(d) and this also compares well with the data in panel (a). Energy scans near the softening wave vector are in good agreement between the data and model [see Fig. 4(a), magenta filled symbols and line]. Figure 2(c) shows also the limitations of the present model. The energy scan shown cuts across the low- $Q$ dispersion and the model (solid line) reproduces well the observed peak position. However, the linewidth is broader than expected based on resolution effects alone (horizontal bar) and there is considerable additional continuum scattering intensity at higher energies above $3 \mathrm{meV}$, which we attribute to multitriplon scattering events, not included in the present model.

With the model parameters kept fixed by the fits to zerofield data, we now calculate the expected behavior in an external magnetic field, which contributes additional terms to the single-ion Hamiltonian; $\mu_{B} \boldsymbol{B} \cdot(\boldsymbol{L}+2 \boldsymbol{S})+12 J_{2} \boldsymbol{S} \cdot\langle\boldsymbol{S}\rangle$. The first term is the Zeeman energy in field and the second term includes the effect of the exchange interactions, treated in a mean-field approximation [29]. $\langle\boldsymbol{S}\rangle$ is the field-induced spin polarization of the ground state, i.e., $\langle\boldsymbol{S}\rangle=\left\langle\psi_{0}|\boldsymbol{S}| \psi_{0}\right\rangle$, where $\psi_{0}$ is the ground state wave function of the single-site Hamiltonian. Solving for $\langle\boldsymbol{S}\rangle$ self-consistently we find the wave functions $\psi_{1,2,3}$ and energies $\lambda_{1,2,3}$ for a general field direction, determine the triplon dispersion relations and neutron structure factor, then average the spectrum over a spherically uniform distribution of powder grains (see Ref. [12] for details). The model calculations are compared with the measured INS data in Fig. 3, panels (e)-(f) with (b)-(c) at 4 and $8.5 \mathrm{~T}$; the model captures the apparent upwards shift of the scattering intensity upon increasing field. This is even more clearly seen in the energy scans in Fig. 4(a), the model calculations (dashed lines) reproduce well the observed shift of spectral weight to higher energies upon increasing field with no adjustable parameters once the overall intensity scale factor is fixed by the comparison in zero field.

It is insightful to compare the spin-orbital triplons of a SOS ground state discussed here with triplons of a spinsinglet (SS) ground state with a completely quenched orbital degree of freedom, as found, for example, in quantum dimerized antiferromagnets like $\mathrm{TlCuCl}_{3}$ [30]. For the latter, a magnetic field Zeeman splits the triplet into $S_{z}=-1,0,+1$ states, with a linear reduction in the gap to the $S_{z}=-1$ state, as shown schematically in Fig. 4(c). At a critical field level crossing with the ground state occurs and magnetic order ensues via condensation of triplons. One might wonder how the behavior of spin-orbital triplons can be any different; the triplons now have an effective angular momentum $J_{\text {eff }}=1$ (as opposed to $S=1$ in the SS case). A low applied magnetic field Zeeman splits the triplet into $J_{\mathrm{eff}, z}=-1,0,+1$ states [4,23]; however, at higher fields terms quadratic and higher in $B$ prevail [21,31] and allow mixing between the SOS and the $J_{\text {eff }, z}=0$ triplet mode. This enables the ground state to reduce its energy in applied field by acquiring a finite polarization along the field direction, see Fig. 4(b), thus avoiding magnetic order via level crossing with the triplet states.

We now relate our results to the generic phase diagram in Fig. 1, describing the transition from SOS to magnetic or orbital order upon increasing $x=J_{2} / \lambda$. Using the parameters obtained from fitting the INS data yields $x \simeq 0.060$, marginally close to the proposed critical value $x_{c}=1 / 16$. For such close proximity to criticality one might expect manifestations of enhanced quantum fluctuations associated with the critical point. For $x \lesssim x_{c}$, in addition to sharp triplon excitations, one would also expect multitriplon continua at higher energies, with enhanced spectral weight and decreasing gap as $x \nearrow x_{c}$, with the triplon dispersions becoming lower boundaries of a critical continuum of excitations precisely at the quantum critical point at $x_{c}$. Effects associated with such continuum scattering and/or broadening of sharp modes may be at least partly responsible for the extra scattering intensity and broadening effects observed in the INS data in Fig. 2(c); we hope our results will stimulate further theoretical modeling of such effects close to spin-orbital quantum criticality.

One may ask if other materials may exhibit related physics. We note that a high-spin $d^{4}$ ion (e.g., $\mathrm{Mn}^{3+}$ ) in an octahedral (weak) cubic crystal field displays the same single-ion physics (electron analogue) as $\mathrm{Fe}^{2+}$ in $\mathrm{FeSc}_{2} \mathrm{~S}_{4}$, i.e., spin $S=2$ and twofold orbital degeneracy, where the spin-orbit coupling (now $\lambda_{0}>0$ ) stabilizes the SOS ground state in Eq. (1) with a $J_{\text {eff }}=1$ excited triplet. Similar singlet-triplet physics, but with a singlet ground state distinct from Eq. (1), originating from $S=1$ and threefold orbital degeneracy, is expected for low-spin $d^{4}$ ions (e.g., $\left.\mathrm{Ru}^{4+}\right)$ in strong octahedral crystal field $[2,5]$ and $d^{8}$ ions (e.g., $\mathrm{Ni}^{2+}$ ) in the tetrahedral field [32]. If such ions can resist JT distortions, they are candidates to display correlated spin-orbit states under intersite exchange, potentially in a different part of the phase diagram in Fig. 1.

To summarize, we have reported powder INS measurements of the full bandwidth of magnetic excitations in the spinel $\mathrm{FeSc}_{2} \mathrm{~S}_{4}$ and have found that the key dispersive features can be well described by spin-orbital triplons of a near-critical SOS state. In high applied magnetic field we have observed a shift of spectral weight to higher energies, giving support to the theoretical proposal [29] that applied fields further stabilize the SOS state by moving the system away from the quantum critical point, this is a direct consequence of the entangled spin-orbital nature of the ground and excited triplet states.

In accordance with the EPSRC policy framework on research data, access to the data will be made available from Ref. [33]. 
This work was partially supported by the EPSRC (UK) under Grants No. EP/H014934/1 and EP/M020517/1, as well as the SNF SCOPES project IZ73Z0_152734/1, the Marie Curie FP7 COFUND PSI Fellowship program, Swiss National Science Foundation, Sinergia Network Mott Physics Beyond the Heisenberg Model, the ERC Grant Hyper Quantum Criticality (HyperQC) and Transregional Research Collaboration TRR 80 (Augsburg, Munich, Stuttgart). This work is partially based on experiments performed at the Swiss spallation neutron source SINQ, Paul Scherrer Institute, Villigen, Switzerland.

Note added.-As this work was being completed Ref. [34] appeared, reporting evidence for marginal magnetic order in samples synthesized using a different protocol, suggesting an extreme sensitivity to the synthesis route. Broadly speaking, there are three main physical factors that could lead to such a discrepancy: vacancies, site disorder, and off-stoichiometry, all of which are discussed in the Supplemental Material [12]. We conclude that offstoichiometry can lead to magnetic order with a transition temperature of a few $\mathrm{K}$. Our results highlighting that magnetic fields favor the SOS state suggest a very interesting possibility that fields applied onto an ordered sample, potentially along a particular direction in a single crystal, may drive it towards the SOS state and thus reach the long-searched-for quantum critical point.

[1] G. Chen, L. Balents, and A. P. Schnyder, Phys. Rev. Lett. 102, 096406 (2009).

[2] G. Khaliullin, Phys. Rev. Lett. 111, 197201 (2013).

[3] H. A. Jahn and E. Teller, Proc. R. Soc. A 161, 220 (1937).

[4] W. Low and M. Weger, Phys. Rev. 118, 1119 (1960).

[5] A. Jain, M. Krautloher, J. Porras, G. H. Ryu, D. P. Chen, D. L. Abernathy, J. T. Park, A. Ivanov, J. Chaloupka, G. Khaliullin, B. Keimer, and B. J. Kim, arXiv:1510.07011.

[6] L. Mittelstädt, M. Schmidt, Z. Wang, F. Mayr, V. Tsurkan, P. Lunkenheimer, D. Ish, L. Balents, J. Deisenhofer, and A. Loidl, Phys. Rev. B 91, 125112 (2015).

[7] V. Fritsch, J. Hemberger, N. Büttgen, E.-W. Scheidt, H. A. Krug von Nidda, A. Loidl, and V. Tsurkan, Phys. Rev. Lett. 92, 116401 (2004).

[8] A. Krimmel, M. Mücksch, V. Tsurkan, M. M. Koza, H. Mutka, and A. Loidl, Phys. Rev. Lett. 94, 237402 (2005).

[9] P. A. Tomas and M. Guittard, Matt. Res. Bull. 14, 249 (1979).

[10] N. Büttgen, A. Zymara, C. Kegler, V. Tsurkan, and A. Loidl, Phys. Rev. B 73, 132409 (2006).

[11] R. Bewley, R. Eccleston, K. McEwen, S. Hayden, M. Dove, S. Bennington, J. Treadgold, and R. Coleman, Physica (Amsterdam) 385B-386B, 1029 (2006).

[12] See Supplemental Material at http://link.aps.org/ supplemental/10.1103/PhysRevLett.118.067205 for details of the single ion and spin-orbital triplon calculations, as well as a description of the background subtraction procedure and information on sample preparation and characterization, which includes Refs. [13-20].
[13] M. Hutchings, Solid State Phys. 16, 227 (1964).

[14] C. Testelin, C. Rigaux, A. Mauger, A. Mycielski, and C. Julien, Phys. Rev. B 46, 2183 (1992).

[15] B. Grover, Phys. Rev. 140, A1944 (1965).

[16] R. M. White, M. Sparks, and I. Ortenburger, Phys. Rev. 139, A450 (1965).

[17] A. G. D. Maestro and M. J. P. Gingras, J. Phys. Condens. Matter 16, 3339 (2004).

[18] G. Squires, Introduction to the Theory of Thermal Neutron Scattering (Cambridge University Press, Cambridge, England, 1978).

[19] P. Fischer, G. Frey, M. Koch, M. Konnecke, V. Pomjakushin, J. Schefer, R. Thut, N. Schlumpf, R. Burge, U. Greuter, S. Bondt, and E. Berruyer, Physica (Amsterdam) 276B-278B, 146 (2000).

[20] V. Tsurkan, A. Loidl et al. (to be published).

[21] D. Ish and L. Balents, Phys. Rev. B 92, 094413 (2015).

[22] A. Abragam and B. Bleaney, Electron Paramagnetic Resonance of Transition Ions (Dover, New York, 1970).

[23] N. J. Laurita, J. Deisenhofer, L. D. Pan, C. M. Morris, M. Schmidt, M. Johnsson, V. Tsurkan, A. Loidl, and N. P. Armitage, Phys. Rev. Lett. 114, 207201 (2015).

[24] As discussed in Refs. [23,25], the transition from the ground state to the lowest level of the orbital triplet occurs at $\hbar \Omega=\hbar \Omega_{0}-E_{\mathrm{JT}}$, where $E_{\mathrm{JT}}$ is an energy shift due to the coupling to Jahn-Teller phonons. We have used the estimate $E_{\mathrm{JT}} \approx 7 \mathrm{meV}$ [23] to deduce the energy of the purely electronic transition $\hbar \Omega_{0}$ and this was then used in the data parameterization to constrain $B_{4}$ and $\lambda_{0}$.

[25] S. Wittekoek, R. P. van Stapele, and A. W. J. Wijma, Phys. Rev. B 7, 1667 (1973).

[26] Note that the usually assumed lowest order approximation $\lambda \approx 6 \lambda_{0}^{2} /\left(120\left|B_{4}\right|\right)$ [4] would predict a value $\sim 30 \%$ higher than that obtained from directly calculating the energy levels of the full single-ion Hamiltonian.

[27] S. Sarkar, T. Maitra, R. Valentí, and T. Saha-Dasgupta, Phys. Rev. B 82, 041105 (2010).

[28] L. F. Feiner, J. Phys. C 15, 1515 (1982).

[29] G. Chen, A. P. Schnyder, and L. Balents, Phys. Rev. B 80, 224409 (2009).

[30] C. Rüegg, B. Normand, M. Matsumoto, A. Furrer, D. F. McMorrow, K. W. Krämer, H. U. Güdel, S. N. Gvasaliya, H. Mutka, and M. Boehm, Phys. Rev. Lett. 100, 205701 (2008).

[31] The $g$-factor characterizing the linear splitting is predicted [21] to be wave vector dependent and vary as the square of the zero-field triplon energy in Eq. (2), therefore to become negligibly small near the magnetic softening wave vector, where the regime of linear splitting of the triplet modes is practically unobservable as quadratic and higher order terms in $B$ dominate.

[32] We have explicitly verified by direct calculation of the wave functions that at the single-ion level all the above cases have qualitatively the same behavior in applied field, i.e., the singlet state is stabilized as shown in Fig. 4(b).

[33] Data location TBC, https://ora.ox.ac.uk/objects/uuid: 1c65d1d8-f751-49fa-afe3-10a61ecbb375.

[34] K. W. Plumb, J. R. Morey, J. A. Rodriguez-Rivera, H. Wu, A. A. Podlesnyak, T. M. McQueen, and C. L. Broholm, Phys. Rev. X 6, 041055 (2016). 